VIII ${ }^{\text {ìmes }}$ Journées Nationales Génie Civil - Génie Côtier, Compiègne, 7-9 septembre 2004

\title{
Prédiction de la cinématique de houle en zone de déferlement
}

\author{
Christophe Chevalier ${ }^{(a)}$, Marilyne Luck ${ }^{(b)}$, Michel Benoit ${ }^{(b)}$ \\ (a)Centre Français du Littoral Maison de la Mer, Quai de la Fosse, 44000 NANTES \\ DHI Eau et Environnement1 rue Du Guesclin, 44000 NANTES \\ (b)Laboratoire National d'Hydraulique et Environnement, \\ EDF R\&D 6 quai Watier, BP 49, 78400 CHATOU
}

\section{Résumé}

L'objet de cette communication est la prédiction de la cinématique de la houle en zone côtière, là où les variations de bathymétrie affectent la propagation de la houle et ses caractéristiques. Des mesures de vitesse ont été réalisées d'abord près du fond pour une large gamme de houles pré-déferlantes et déferlantes, puis selon différents profils verticaux dans la zone de déferlement pour trois conditions spécifiques. Ces données expérimentales sont utilisées pour évaluer et comparer les prédictions données par différents modèles numériques parmi les théories à fond plat, les modèles à fond incliné et les méthodes de fonction de transfert. Ces dernières donnent les meilleures prédictions si le profil local de surface libre est connu. Certains modèles à fond incliné peuvent également donner des prédictions fiables à partir d'un nombre limité de paramètres de houle. Les théories à fond plat ne sont pas recommandées en zone côtière.

\begin{abstract}
This paper deals with the prediction of wave kinematics in the nearshore and coastal zones, where bottom variations strongly affect wave propagation and characteristics. Measurements of wave kinematics have been performed near the bottom for a large number of shoaling and breaking wave conditions, and then all over the water column inside the breaking zone for three particular conditions. These experimental data serves to evaluate and compare several numerical prediction methods, including Flat Bottom Theories (FBT), Sloping Bottom Methods (SBM) and Transfer Function Methods (TFM). It is shown that advanced TFM give the best predictions for wave kinematics, provided that the local free surface elevation signal is available. Particular SBM may also produce reliable prediction for the case of plane slope from a limited number of wave parameters, whereas FBT should not be used in the coastal zone.
\end{abstract}

\section{Introduction}

La connaissance de la cinématique de la houle est très importante pour de nombreux problèmes d'ingénierie offshore et côtière (dimensionnement de structures, transport sédimentaire...). Afin d'évaluer différentes stratégies et méthodes de prédiction des vitesses issues de la littérature ( $(2)$, nous avons mené une étude comparative de celles-ci sur une large gamme de conditions de houle. Ceci a nécessité la mise en place d'une procédure expérimentale spécifique, notamment pour l'évaluation quantitative et objective des différentes prédictions cinématiques $(\S 3)$.

Une première étape de validation concerne la prédiction de la vitesse horizontale près du fond pour un grand nombre de conditions de houle (§ 4). 
La deuxième étape de l'analyse s'intéresse plus spécifiquement à la dépendance verticale des vitesses orbitales horizontale et verticale dans la zone de déferlement (§5). Les prédictions des modèles cinématiques sont ainsi comparées à des mesures détaillées de vitesse selon des profils verticaux dans la zone de déferlement pour trois conditions spécifiques.

\section{Méthodes de prédiction de la cinématique de houle}

La détermination de la cinématique des vagues nécessite un certain nombre d'informations sur la houle et la bathymétrie. Une classification des différentes stratégies de prédiction a été introduite par Benoit et al. . Elle distingue cinq classes principales, en fonction d'une part du niveau d'information disponible sur les conditions de houle et la bathymétrie, et d'autre part des hypothèses et bases théoriques sur lesquelles reposent les modèles de prédiction (voir figure 1).

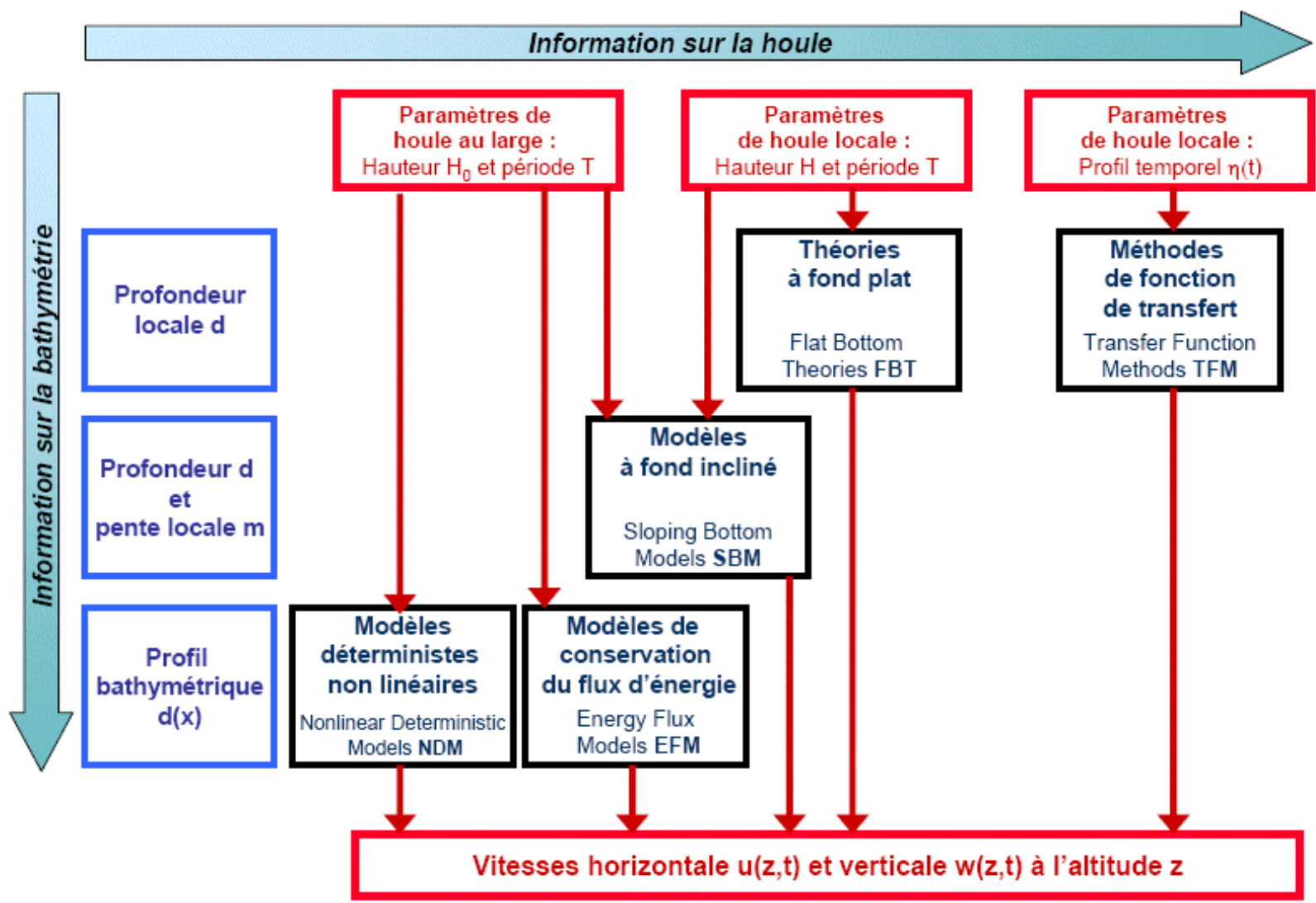

Figure 1 : Classification des méthodes et théories permettant d'obtenir la cinématique de houle en zone côtière (d'après Benoit et al. ${ }^{1}$ )

Dans la présente étude, on s'intéresse plus particulièrement aux classes FBT, SBM et TFM pour lesquelles une connaissance des conditions locales est nécessaire. Les modèles des deux autres classes (EFM et NDM) appartiennent plus généralement à la catégorie des modèles de propagation de houle, dont l'évaluation est plus complexe et n'est pas abordée à ce stade de notre étude. Les théories fond plat (FBT) ont été développées pour des houles se propageant sans déformation sur un fond plat. On retrouve dans cette catégorie les théories analytiques de type 
Stokes et cnoïdales et le modèle numérique basé sur la décomposition de la fonction de courant en série de Fourier, présenté par Dean .

Les modèles à fond incliné (SBM) sont soit des extensions des théories de houle à fond plat, soit des modèles semi-empiriques visant à reproduire certains des effets liés à la propagation des vagues sur un fond de pente $\mathrm{m}$, supposée constante. En particulier, ces modèles tentent de reproduire la dissymétrie de la houle par rapport à un plan vertical. La méthode empirique de Isobe et Horikawa et sa variante proposée par Grasmeijer et van Rijn sont deux modèles de cette classe. Lorsque le profil de surface libre est connu, il est possible d'estimer la cinématique de houle au droit de ce profil par différentes méthodes utilisant soit des fonctions de transfert directes et empiriques telles que la méthode de la fonction de transfert modifiée (Koyama et Iwata ), soit des modèles numériques plus élaborés, comme la forme irrégulière de la fonction de courant (Dean ). Le tableau 1 récapitule les caractéristiques des différents modèles cinématiques retenus pour évaluation dans cette communication.

Tableau 1 : Récapitulatif des modèles cinématiques étudiés.

\begin{tabular}{|c|c|c|c|c|}
\hline Sigle & Modèle & Classe & Entrée & Sortie \\
\hline CNO3 & \begin{tabular}{ll}
\multicolumn{1}{c}{ Théorie } & $\begin{array}{l}\text { cnoïdale } \\
\text { (d'après } \\
\text { d'ordre } 3\end{array}$ \\
Horikawa6) & \\
\end{tabular} & FBT & $\mathrm{H}, \mathrm{T}, \mathrm{d}$ & $\begin{array}{r}\eta(\mathrm{t}) \\
\mathrm{u}(\mathrm{z}, \mathrm{t}) \\
\mathrm{w}(\mathrm{z}, \mathrm{t})\end{array}$ \\
\hline DSFM & $\begin{array}{l}\text { Forme régulière de la } \\
\text { fonction de courant } \\
\text { (Dean2) }\end{array}$ & FBT & $\mathrm{H}, \mathrm{T}, \mathrm{d}$ & $\begin{array}{r}\eta(\mathrm{t}) \\
\mathrm{u}(\mathrm{z}, \mathrm{t}) \\
\mathrm{w}(\mathrm{z}, \mathrm{t})\end{array}$ \\
\hline IH82 & $\begin{array}{l}\text { Méthode de Isobe et } \\
\text { Horikawa3 }\end{array}$ & SBM & $\mathrm{m}_{\mathrm{H}}^{\mathrm{H}, \mathrm{T}, \mathrm{d},}$ & $\mathrm{u}(\mathrm{z}, \mathrm{t})$ \\
\hline IH98 & $\begin{array}{l}\text { Modification de IH82 } \\
\text { par Grasmeijer et van } \\
\text { Rijn4 }\end{array}$ & SBM & $\mathrm{m}_{\mathrm{H}} \mathrm{H}, \mathrm{T}, \mathrm{d}$, & $\mathrm{u}(\mathrm{z}, \mathrm{t})$ \\
\hline MTFM & $\begin{array}{l}\text { Modèle de Koyama et } \\
\text { Iwata5 }\end{array}$ & TFM & $\begin{array}{ll}\mathrm{d} & \eta(\mathrm{t}), \mathrm{T},\end{array}$ & $\begin{array}{r}\mathrm{u}(\mathrm{z}, \mathrm{t}), \\
\mathrm{w}(\mathrm{z}, \mathrm{t})\end{array}$ \\
\hline ISFM & $\begin{array}{l}\text { Forme irrégulière de } \\
\text { la fonction de courant } \\
\text { (Dean2) }\end{array}$ & TFM & $\mathrm{d}^{\eta(\mathrm{t}),}, \mathrm{T}$, & $\begin{array}{r}\mathrm{u}(\mathrm{z}, \mathrm{t}), \\
\mathrm{w}(\mathrm{z}, \mathrm{t})\end{array}$ \\
\hline
\end{tabular}

\section{Méthodologie de validation expérimentale}

\section{1.Étude expérimentale en canal à houle}

La partie expérimentale de cette étude a été menée en canal à houle (longueur $22 \mathrm{~m}$, largeur $0.8 \mathrm{~m}$, hauteur d'eau $0.5 \mathrm{~m}$ ) à l'Université de Caen. Une plage de $10 \mathrm{~m}$ de longueur et de pente constante $\mathrm{m}=1: 20$, est installée à l'extrémité du canal afin de simuler la déformation de la houle lors de sa propagation vers la côte. Pour mesurer la déformation de la surface libre sur la pente, neuf sondes de surface libre (notées P1 à P9) ont été installées avec un espacement horizontal régulier de $50 \mathrm{~cm}$, entre les profondeurs de 0.3 et $0.1 \mathrm{~m}$. Ces données de surface libre ont été complétées par des mesures de vitesse par vélocimétrie laser soit près du fond, soit selon 
différents profils verticaux dans la zone de déferlement. Les données expérimentales (surface libre et vitesses correspondantes) ont été analysées en utilisant la technique de la vague moyenne. Les grandeurs caractéristiques de surface libre (hauteur et période ou profil temporel) sont ensuite utilisées en entrée des différents modèles cinématiques étudiés. La technique de vague moyenne par phase permet de décomposer chaque vitesse en une composante moyenne permanente umoy, une composante purement orbitale uw, dépendant de la seule phase adimensionnelle $\mathrm{t} / \mathrm{T}$, et une fluctuation turbulente u'. Dans le cas de la vitesse horizontale, cette décomposition s'écrit :

$$
\begin{aligned}
& \mathrm{u}(\mathrm{t})=\mathrm{u}_{\text {moy }}+\mathrm{u}_{\mathrm{w}}(\mathrm{t} / \mathrm{T})+\mathrm{u}^{\prime}(\mathrm{t}) \\
& \mathrm{u}_{\text {moy }}=\frac{1}{\mathrm{~T}} \int_{0}^{\mathrm{T}} \mathrm{u}(\mathrm{t} / \mathrm{T}) \mathrm{dt}
\end{aligned}
$$

La figure 2 présente un exemple de profil temporel de la vitesse mesurée $u(t)$ et de la composante orbitale correspondante uw(t). Les différents modèles évalués dans cette étude ne peuvent rendre compte de la présence du fort courant de retour observé en zone de déferlement, comme l'illustre la comparaison avec la prédiction cinématique donnée par le modèle ISFM sur la figure 2. Par la suite, seule la composante purement orbitale est donc retenue pour comparaison avec les différents modèles cinématiques de prédiction.

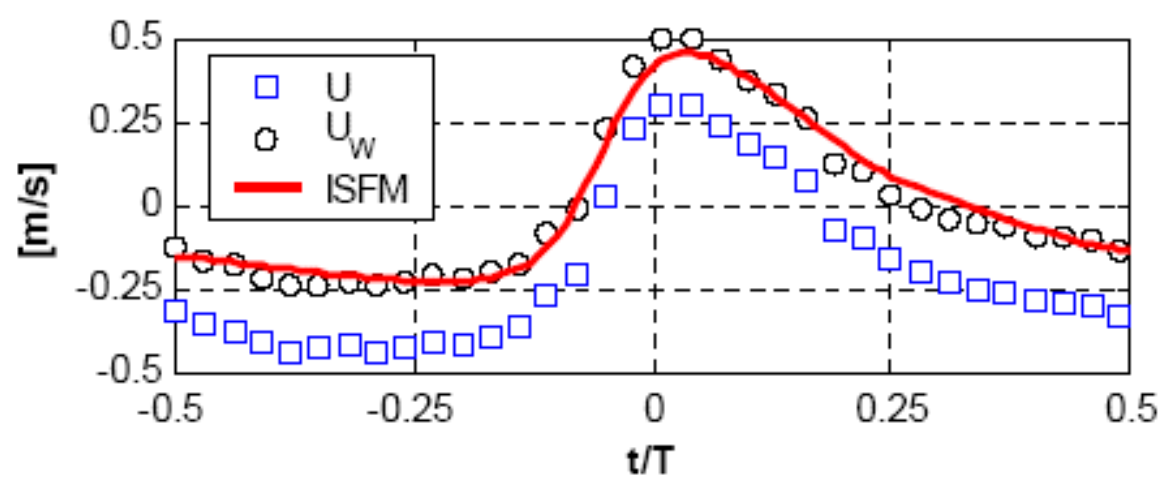

Figure 2 : Exemple de séparation entre mouvement moyen et mouvement purement orbital et comparaison avec la prédiction donnée par le modèle ISFM.

\section{2.Évaluation des performances des modèles cinématiques}

La détermination de la validité d'une prédiction à l'aide d'un graphique tel que celui présenté sur la figure 2 n'est pas satisfaisante, surtout dans le cas de notre étude où les nombres de modèles évalués et de conditions expérimentales étudiées sont élevés. Afin de fournir une évaluation plus objective, nous avons suivi la démarche proposée par Hattori pour quantifier la capacité d'une prédiction numérique temporelle (yi) à reproduire un signal mesuré (xi). Quatre paramètres d'évaluation sont ainsi définis par l'auteur, parmi lesquels nous avons retenu pour 
présentation ici la seule erreur quadratique moyenne ET, définie comme suit :

$$
\mathrm{E}_{\mathrm{T}}(\mathrm{x})=\sqrt{\sum\left(\mathrm{x}_{\mathrm{i}}-\mathrm{y}_{\mathrm{i}}\right)^{2} / \sum \mathrm{x}_{\mathrm{i}}^{2}}
$$

Ce paramètre reflète l'ajustement général entre les deux séries temporelles. Des valeurs indicatives pour apprécier les performances d'une prédiction sont données dans le tableau 2. Toutefois, il convient de noter que ce paramètre est mis en défaut pour les signaux de très faible amplitude (le dénominateur tendant alors vers 0 ), ce qui est notamment le cas pour la vitesse verticale près du fond.

Tableau 2: Limites caractéristiques indicatives du paramètre d'erreur ET

\begin{tabular}{|c|c|r|c|c|c|}
\hline Performance & Excellente & Bonne & Acceptable & Médiocre & Mauvaise \\
\hline ET & $0-0.1$ & $0.1-0.25$ & $0.25-0.5$ & $0.5-1$ & $>1$ \\
\hline
\end{tabular}

\section{Validité des prédictions prés du fond}

\subsection{Présentation}

La première étape de notre étude de validité des prédictions de différents modèles cinématiques a été menée sur un grand nombre de mesures vélocimétriques près du fond (Chevalier et al. ). Différentes hauteurs de houle ont été simulées pour huit périodes différentes (comprises entre 1 et $2.8 \mathrm{~s}$ ). Des mesures de vitesse à $2 \mathrm{~cm} \mathrm{du}$ fond ont été réalisées à trois profondeurs d'eau distinctes $(25,20$ et $15 \mathrm{~cm})$. L'ensemble de ces données représente au total 178 cas-tests qui ont été ensuite comparés aux estimations données par les six modèles cinématiques retenus (cf. Tableau 1). La figure 3 présente ainsi l'étendue des conditions de houle étudiées en fonction des paramètres adimensionnels de houle $(\mathrm{d} / \mathrm{L} 0, \mathrm{H} / \mathrm{L} 0)$ où $\mathrm{L} 0=\mathrm{gT}^{2} /(2 \pi)$, en distinguant également les conditions avant déferlement (graphe de gauche) de celles au point de déferlement ou après (graphe de droite). La figure 3 présente par ailleurs l'évolution du courant permanent umoy à $2 \mathrm{~cm}$ du fond. Sur le graphe de gauche (vagues non déferlantes), on note une très légère augmentation de celui-ci (en valeur absolue) lorsqu'on approche la hauteur limite de déferlement $\mathrm{Hb}$. Pour les vagues déferlantes et post-déferlantes, le courant de retour est plus important : plus on progresse dans la zone de déferlement (la hauteur de houle relative H/L0 diminuant alors), plus le courant de retour est de forte amplitude. 

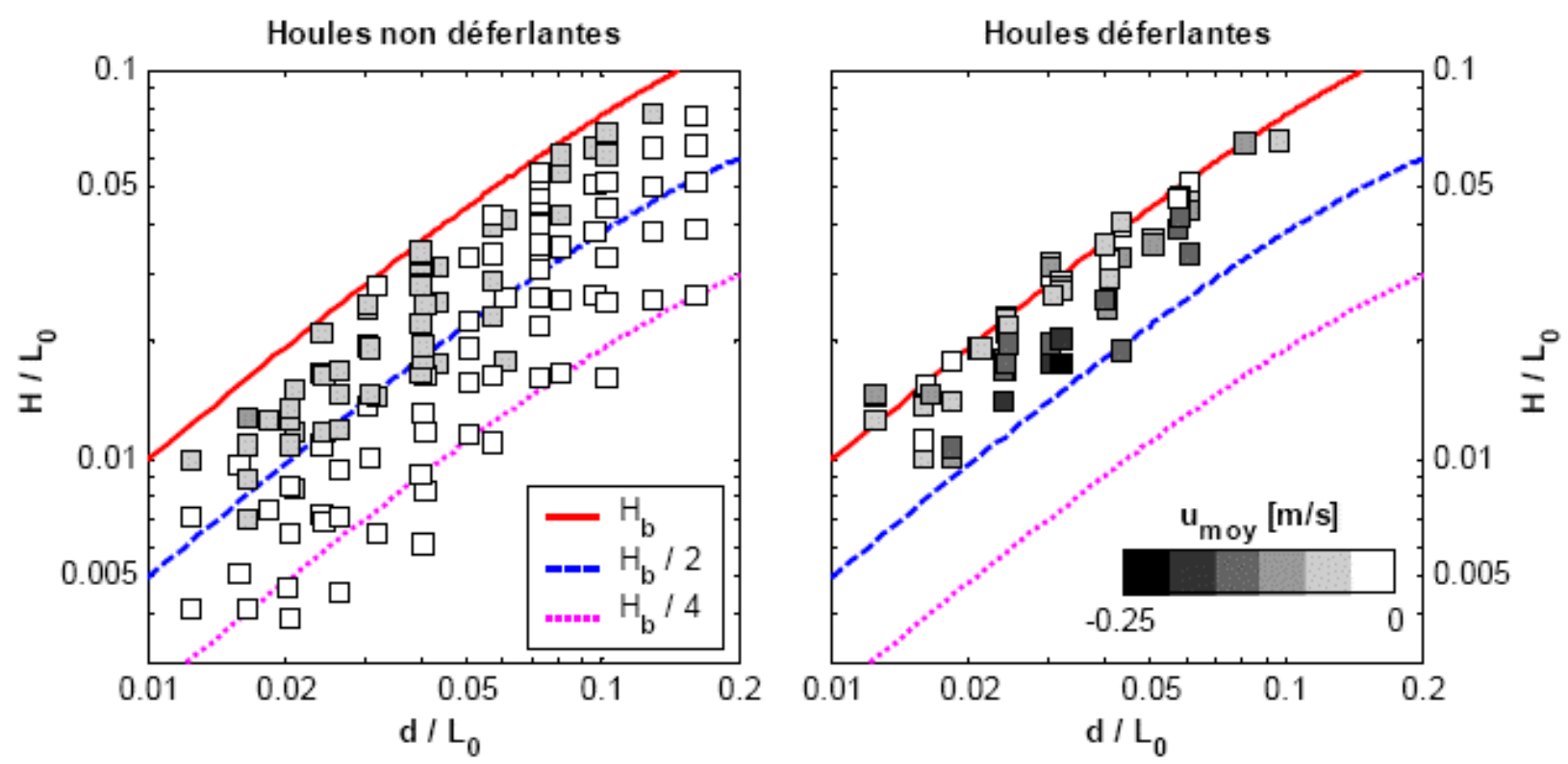

Figure 3 : Conditions expérimentales et intensité du courant de retour umoy (en $\mathrm{m} / \mathrm{s})$ pour les mesures de vitesse horizontale près $d u$ fond

\subsection{Résultats et discussion}

La figure 4 présente pour chacun des modèles de prédiction l'évolution du paramètre ET(uw) en fonction du nombre d'Ursell de faible profondeur (US $=\mathrm{gHT}^{2} / \mathrm{d}^{2}$ ) et le tableau 3 reprend les grandeurs caractéristiques de ce même paramètre sur l'ensemble des données étudiées. A noter que la comparaison est ici effectuée sur la seule composante horizontale de vitesse, la verticale étant négligeable près du fond. L'ensemble de ces éléments permet de proposer un premier classement des différents modèles par ordre décroissant de performance : ISFM, MTFM, IH98, IH82, DSFM et CN03. On note toutefois un regroupement des performances par classe de modèle (TFM, SBM et FBT), malgré des approches de modèlisation parfois très éloignées entre deux modèles d'une même classe ( voir notament les performances des modèles MTFM et ISFM de la classe TFM). Ainsi, plus le niveau d'information requis par le modèle est élevé, meilleure est la prédiction. 

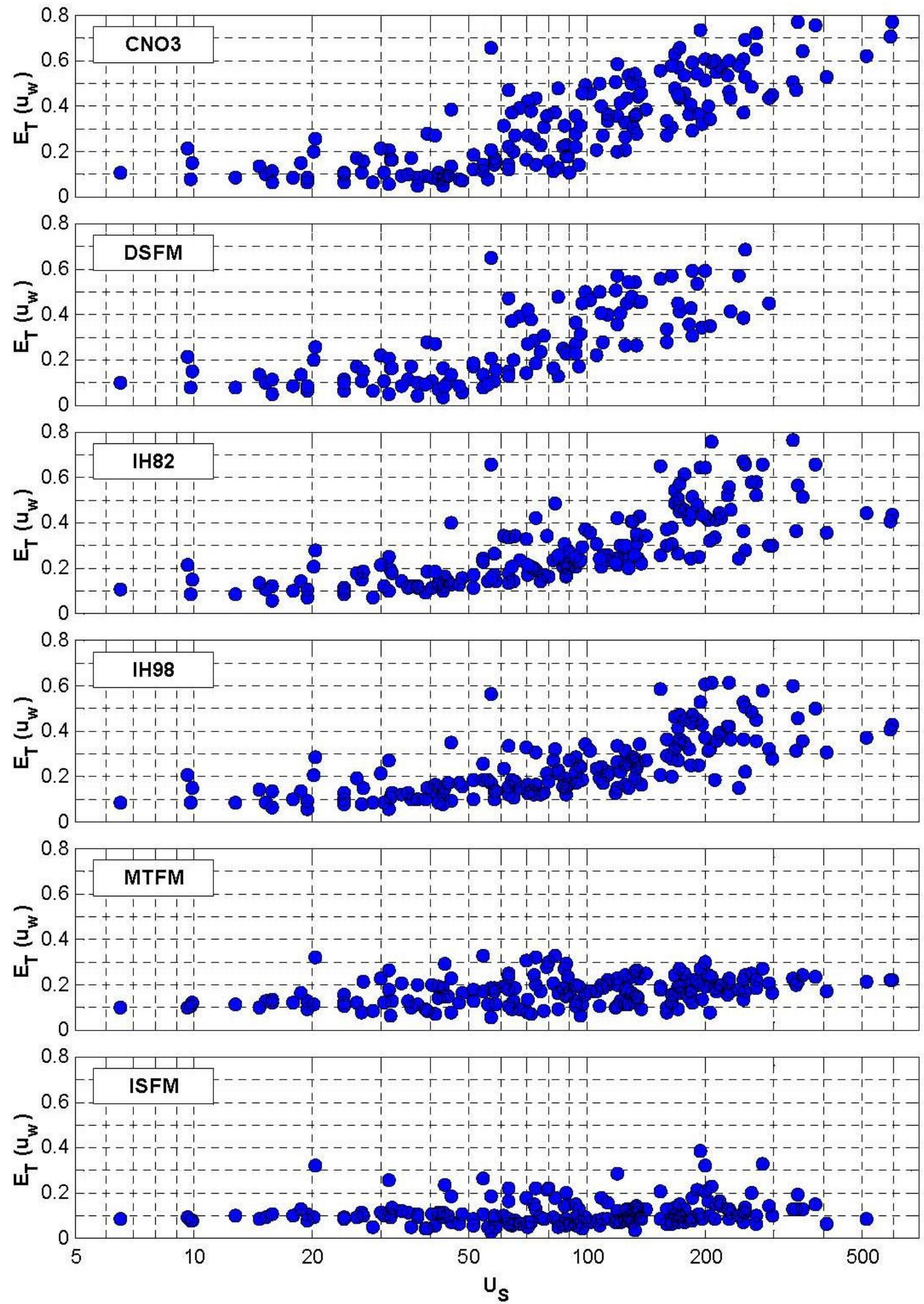

Figure 4: Evolution du paramètre Et( $\mu w)$ en fonction du nombre d'Ursell de faible profondeur Us pour les différents modèles de prédiction. 
L'évolution des performances d'un modèle en fonction du nombre d'Ursell montre également que celles-ci se dégradent généralement lorsque US augmente. Cela est tout particulièrement vrai pour les modèles des classes FBT et SBM, les performances des modèles de la classe TFM restant très satisfaisantes sur l'ensemble des données étudiées.

Tableau 3 : Statistiques sur le paramètre ET(uw) pour les six modèles de prédiction sur l'ensemble des mesures près du fond.

\begin{tabular}{|c|c|c|c|c|c|c|c|}
\hline \multicolumn{2}{|c|}{ Modèle } & CNO3 & DSFM & IH82 & IH98 & MTFM & ISFM \\
\hline \multicolumn{2}{|c|}{ Convergence } & $100 \%$ & $67 \%$ & $\begin{array}{c}100 \\
\%\end{array}$ & $\begin{array}{c}100 \\
\%\end{array}$ & $100 \%$ & $\begin{array}{c}100 \\
\%\end{array}$ \\
\hline \multirow{3}{*}{ ET(uw) } & Moyenne & 0.33 & 0.27 & 0.29 & 0.25 & 0.17 & 0.11 \\
\cline { 2 - 8 } & Ecart-type & 0.20 & 0.17 & 0.16 & 0.13 & 0.06 & 0.06 \\
\cline { 2 - 8 } & Maximum & 0.87 & 0.82 & 0.81 & 0.61 & 0.33 & 0.38 \\
\hline
\end{tabular}

On note enfin, qu'à l'exception notable du modèle DSFM, les autres modèles donnent une solution (physiquement plausible) dans tous les cas. La méthode de la forme régulière de la fonction de courant de Dean ne converge en effet pas dans un grand nombre de cas quand le rapport $\mathrm{H} / \mathrm{d}$ dépasse la valeur de 0.78 , limite théorique pour des vagues stables sur fond plat.

\section{$\underline{5 . V a l i d i t e ́ ~ d e s ~ p r e ́ d i c t i o n s ~ s u r ~ l a ~ c o l o n n e ~ d ' e a u ~}$}

\subsection{Présentation}

Trois conditions de houle régulière, conduisant respectivement à un déferlement glissant et deux déferlements plongeants, ont ensuite été étudiées plus en détail (voir tableau 4). Différents profils verticaux de vitesse ont été réalisés au droit des sondes de surface libre P1 à P9 afin d'étudier la dépendance verticale des vitesses. La figure 5 présente l'évolution de la surface libre et la grille de mesures étudiée pour le cas HR1. A noter qu'à ce stade de l'étude, seules les vitesses mesurées en dessous du creux de la vague sont étudiées et comparées aux prédictions des différents modèles cinématiques.

Tableau 4 :Conditions expérimentales et caractéristiques du déferlement.

\begin{tabular}{|c|c|c|c|c|}
\hline Essai & Période T & $\begin{array}{c}\text { Hauteur } \\
\text { incidente H0 }\end{array}$ & $\begin{array}{c}\text { Type de } \\
\text { déferlement }\end{array}$ & $\begin{array}{c}\text { Point de } \\
\text { déferlement }\end{array}$ \\
\hline HR1 & $1.33 \mathrm{~s}$ & $0.176 \mathrm{~m}$ & Glissant & P3 \\
\hline HR2 & $2.00 \mathrm{~s}$ & $0.152 \mathrm{~m}$ & Plongeant & P4 \\
\hline HR3 & $2.00 \mathrm{~s}$ & $0.192 \mathrm{~m}$ & Plongeant & P3 \\
\hline
\end{tabular}




\subsection{Présentation des résultats et discussion}

Les figures 6.a et 7.a présentent, respectivement pour les essais HR1 et HR3, les profils temporels de surface libre à chaque section de mesure, ainsi que les profils verticaux des vitesses moyennes correspondantes (figures 6.b et 7.b). Les profils verticaux des erreurs quadratiques moyennes ET(uw) et ET(ww) calculées pour chacun des six modèles cinématiques sont également tracés sur les graphes 6.c et 7.c, chaque rangée correspondant respectivement à l'un des modèles du tableau 1. A noter que les résultats pour l'essai HR2, non présentés dans ce papier, sont sensiblement équivalents à ceux obtenus pour l'essai HR3.

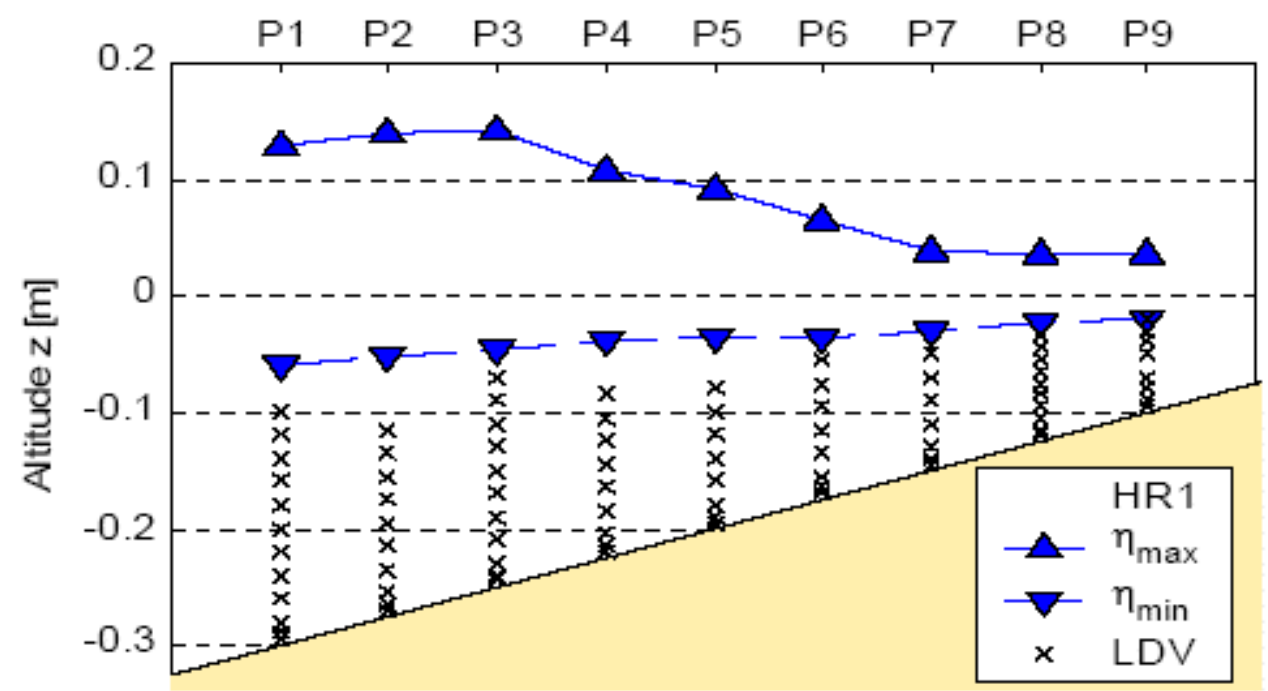

Figure 5: Evolution de la surface et grille de mesures vélocimétriques pour le cas HR1

L'analyse des figures 6.c et 7.c montre que la théorie cnoïdale d'ordre 3 converge dans la plupart des cas, excepté dans la partie proche du creux de la vague (voir par exemple les profils autour et après le point de déferlement de l'essai HR3). Dans ces conditions, une solution est obtenue numériquement, mais elle est en fait physiquement irréaliste (présence des crêtes secondaires). Les estimations données par ce modèle sont correctes lorsque le profil de surface libre reste à peu près symétrique par rapport à un plan vertical (ce qui est quasiment le cas pour les profils $\mathrm{P} 1$ à $\mathrm{P} 3$ de chaque essai). Dans le cas du déferlement glissant, les prédictions restent d'ailleurs acceptables après le point de déferlement (profils P4 à P6), mais les performances se dégradent sur les profils suivants, la dissymétrie entre profil montant et profil descendant étant de plus en plus marquée sur la surface libre et les vitesses au fur et à mesure que la profondeur décroît. Comme pour l'étape de validation précédente, la méthode de la forme régulière de la fonction de courant de Dean (DSFM) ne converge pas dans un grand nombre de cas autour du point de déferlement. En particulier, peu d'estimations peuvent être obtenues par ce modèle pour le cas HR3. Dans les cas de convergence, les résultats obtenus par DSFM sont comparables à ceux obtenus par le modèle $\mathrm{CNO} 3$ et ce pour les vitesses horizontale et verticale. A noter également que les solutions données par DSFM sont toujours physiquement plausibles sur toute la colonne d'eau (contrairement donc à certaines fournies par CNO3). Rappelons que les deux modèles à fond incliné envisagés dans cette étude (IH82 et IH98) ne fournissent une estimation que de la vitesse horizontale. Leurs performances (en terme de paramètre ET), présentées sur les 
rangées 3 et 4 des figures 6.c et 7.c, montrent des résultats très satisfaisants dans la zone de shoaling. Ceci se traduit en effet par des valeurs de ET toujours inférieures à 0.5 (voire 0.25 pour les trois premiers profils de chaque essai). A proximité du point de déferlement et dans la zone de déferlement, la méthode IH82 donne dans l'ensemble une estimation de moins en moins satisfaisante, les meilleures estimations étant obtenues dans le cas du déferlement glissant HR1.

Sous ces mêmes conditions, le modèle modifié IH98, proposé par Grasmeijer et van Rijn , donne très clairement des meilleurs résultats que la méthode originelle IH82. L'erreur ET dépasse ainsi rarement la valeur 0.5 dans tous les essais, confirmant donc tout l'intérêt de cette méthode, particulièrement simple à mettre en œuvre. Le modèle MTFM (Koyama et Iwata ) donne une bonne estimation de la vitesse horizontale dans la zone de déferlement (ET est généralement inférieure à 0.25). Les prédictions sont très clairement meilleures que celles obtenues par les méthodes des classes FBT et SBM considérées plus haut. Le modèle MTFM apparaît donc comme un outil très attractif, et ce d'autant plus qu'il est très simple à mettre en œuvre (à condition de posséder un enregistrement temporel de surface libre). Cependant, l'estimation de la composante verticale est médiocre, le paramètre ET correspondant étant le plus souvent supérieur à 0.5 , y compris avant le point de déferlement. Le modèle ISFM (Dean ) permet également d'obtenir de très bonnes prédictions de la composante orbitale horizontale, prédictions généralement légèrement meilleures que celles données par le modèle MTFM. Pour ces deux méthodes, les prédictions en zone de surf interne sont parfois moins satisfaisantes près du fond et du creux de la vague, mais restent acceptables (ET de l'ordre de 0.5 ou moins). Pour la composante orbitale verticale, les prédictions données par le modèle ISFM sont clairement meilleures que celles données par le modèle MTFM.

Dans les deux cas, on note cependant une augmentation de l'erreur quadratique moyenne correspondante près du fond. Ceci est lié à la faible amplitude du signal mesuré à cet endroit, conduisant à de fortes valeurs non significatives du paramètre ET. Étant donné que le profil temporel de surface libre est utilisé comme paramètre d'entrée du calcul, les bons résultats obtenus par les modèles TFM ne sont pas surprenants (à l'exception de l'estimation de la vitesse verticale donnée par le modèle MTFM). Le fait de prendre en compte le profil dissymétrique par rapport à un plan vertical améliore grandement la qualité des prédictions de la cinématique, particulièrement en zone de déferlement.

\section{Conclusions}

L'objet de cette communication est l'étude de la validité expérimentale de modèles de prédiction de la cinématique en zone côtière. A cet effet, des mesures détaillées de vitesse ont été réalisées en canal à houle, et plus particulièrement dans la zone de déferlement, pour différentes conditions expérimentales. Les prédictions données par les théories de houle à fond plat (FBT : théorie cnoïdale à l'ordre 3 et forme régulière de la théorie de la fonction de courant) sont, sans surprise, médiocres pour des houles en zone côtière. Par ailleurs, les résultats doivent être examinés avec soin, certaines solutions obtenues numériquement pouvant être physiquement irréalistes. Pour des conditions de houle déferlante, les performances des modèles à fond incliné (SBM) sont supérieures à celles de la classe FBT. Le modèle IH82 de Isobe et Horikawa présente ainsi un bon accord avec les mesures de vitesse horizontale avant le point de déferlement, la modification de ce modèle IH98 proposée par Grasmeijer et van Rijn permettant par ailleurs une meilleure prédiction dans la zone de déferlement. Parmi les modèles utilisés, la 
forme irrégulière de la théorie de la fonction de courant (ISFM) présente les meilleures performances pour les vitesses horizontale et verticale sur l'ensemble de la zone de déferlement. La méthode de la fonction de transfert modifiée (MTFM) donne également de bons résultats pour la vitesse horizontale, les résultats obtenus pour la composante verticale n'étant pas satisfaisants. Même si la méthode ISFM apparaît plus précise et puissante, la simplicité de mise en œuvre du modèle MTFM le rend tout particulièrement intéressant lorsque seule la composante horizontale est nécessaire. Il faut toutefois rappeler que ces deux méthodes nécessitent le maximum d'informations sur la houle (profil temporel de surface libre).

\section{Remerciements}

Ce travail a été financé en partie par le Fond de Soutien aux Hydrocarbures (FSH), au travers du projet CLAROM-ECOMAC (contrat 99.4DM06101). Les auteurs remercient également Lars Messutat, élève ingénieur de l'Université de Braunschweig, pour son aide lors de la conduite des essais expérimentaux présentés dans cette publication.

\section{Références}

1.Benoit M., Luck M., Chevalier C., Bélorgey M. (2002). Near-bottom kinematics of shoaling and breaking waves: experimental investigation and numerical prediction. Proc. 28th Int. Conf. on Coastal Eng., Cardiff (UK), pp 306-318.

2.Dean R.G. (1965) Stream function representation of nonlinear ocean waves. J. Geophys. Res., vol. 70, $\mathrm{n}^{\circ} 18$, pp 4561-4572.

3.Isobe M., Horikawa K. (1982) Study on water particle velocities of shoaling and breaking waves. Coastal Eng.in Japan, Vol 25, pp 109-123.

4.Grasmeijer B.T., Van Rijn L.C. (1998). Breaker bar formation and migration. Proc. 26th Int. Conf. on Coastal Eng., Copenhagen (Denmark), pp 2750-2758.

5.Koyama H., Iwata K. (1985) Estimation of water particle velocities by a modified transfer function method. Coastal Eng. in Japan, Vol. 28, pp 1-13.

6.Horikawa K. (1988). Nearshore dynamics and coastal processes. University of Tokyo Press, Tokyo (Japan), $522 \mathrm{p}$.

7.Hattori M. (1986). Experimental study on the validity range of various wave theories. Proc. 20th Int. Conf. on Coastal Eng., Taipei (Tawain), pp 232-246.

8.Chevalier C., Luck M., Benoit M. (2003) Validation expérimentale des méthodes de prédiction de la cinématique de houle - Estimation des vitesses orbitales près du fond en zone côtière. Rapport technique EDF-LNHE, $\mathrm{n}^{\circ} \mathrm{HP}-75 / 03 / 077 / \mathrm{A}$. 


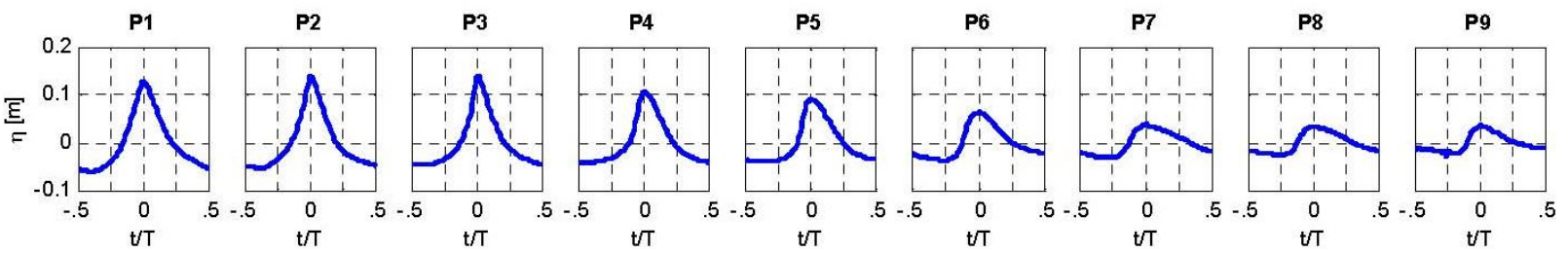

6.a) Évolution du profil temporel de surface libre à chaque section de mesure

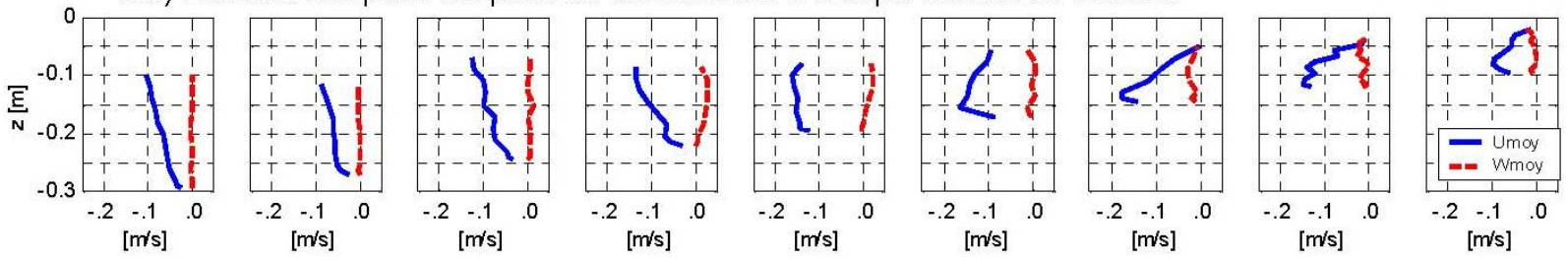

6.b) Profils verticaux des vitesses moyennes $u_{\text {mov }}$ et $w_{\text {mov }}$ (entre le fond et le creux de la vague).

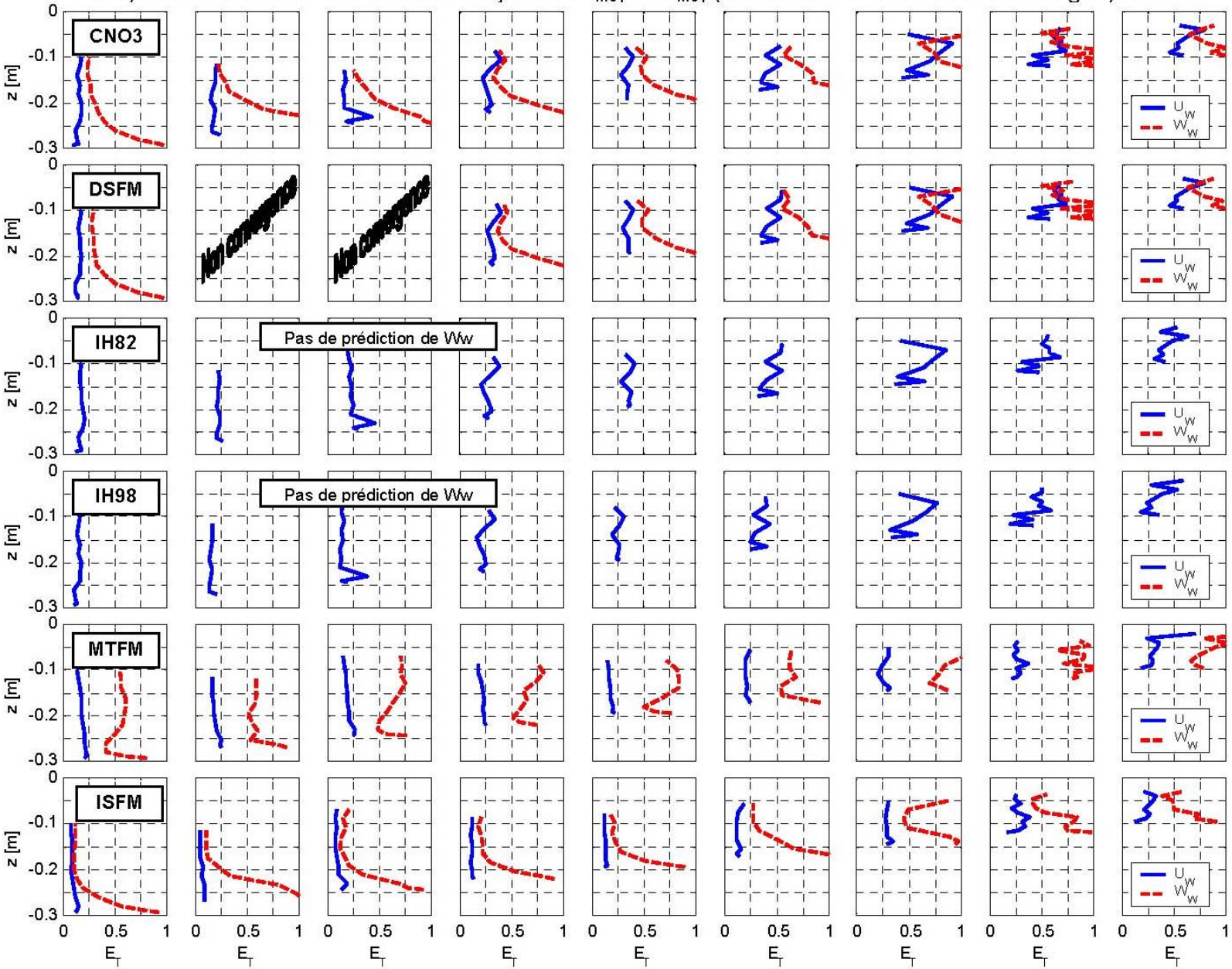

6.c) Profil verticaux de l'erreur quadratique moyenne ET pour estimer $U w(t / T)$ et $W w(t / T)$

Figure 6: Résultat de l'essai $H R 1(H o=17.6 \mathrm{~cm}, T=1.33 \mathrm{~s})$ 


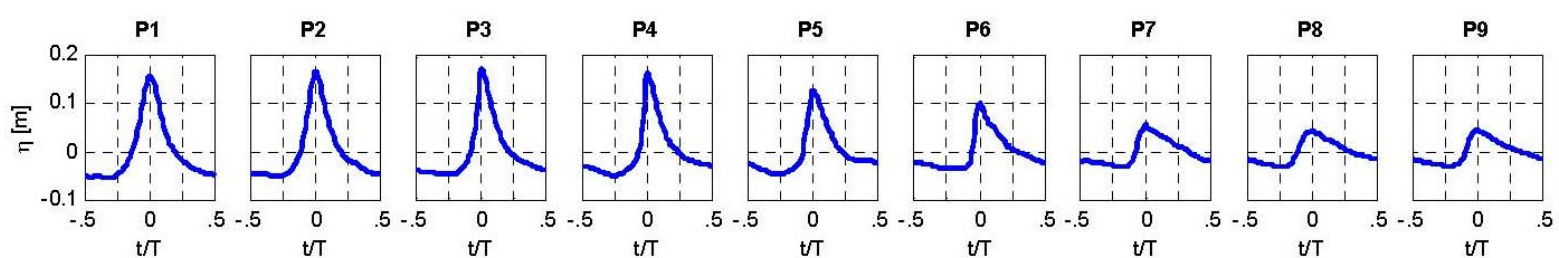

7.a) Évolution du profil temporel de surface libre à chaque section de mesure

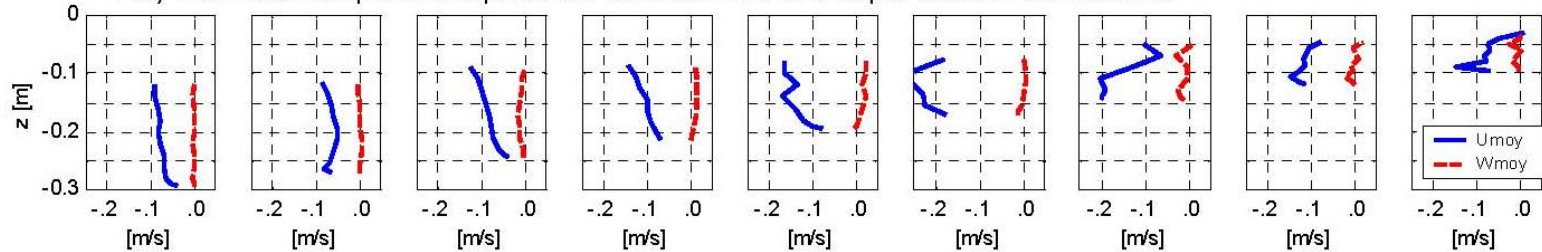

7.b) Profils verticaux des vitesses moyennes $u_{\text {mov }}$ et $w_{\text {mov }}$ (entre le fond et le creux de la vague).

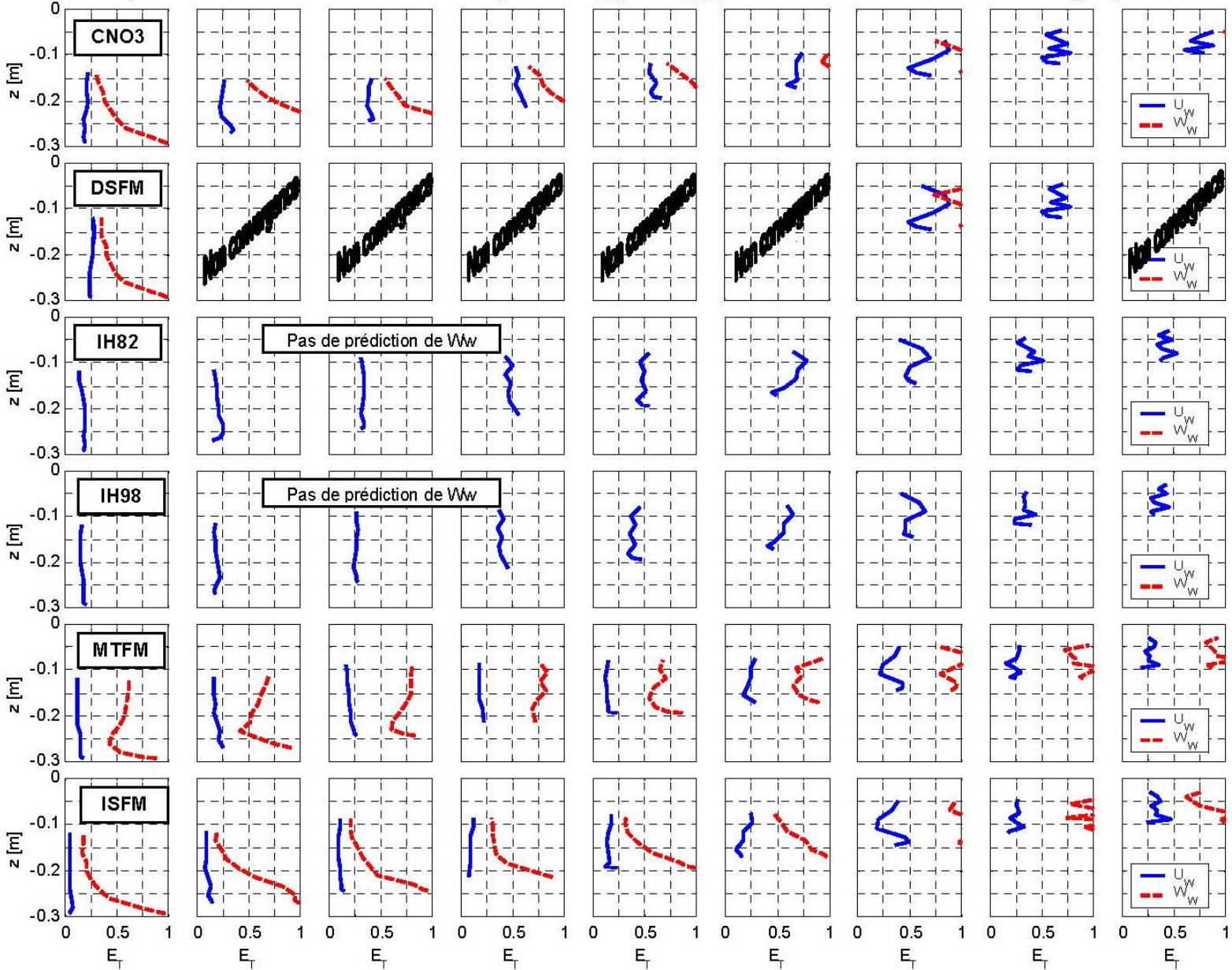

7.c) Profil verticaux de l'erreur quadratique moyenne ET pour estimer $U w(t / T)$ et $W w(t / T)$

Figure 7: Résultat de l'essai $H R 3(H o=19.2 \mathrm{~cm}, T=2 \mathrm{~s})$ 